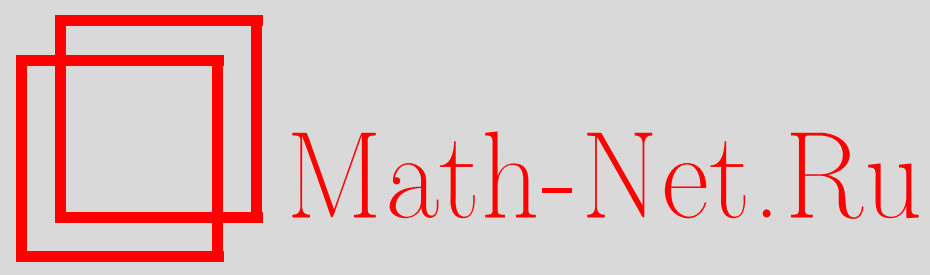

Б. Н. Хабибуллин, Устойчивость полноты экспоненциальных систем на выпуклых компактах в $\mathbb{C}$, Матем. заметки, 2002, том 72, выпуск 4, 587-596

DOI: https://doi.org/10.4213/mzm447

Использование Общероссийского математического портала Math-Net.Ru подразумевает, что вы прочитали и согласны с пользовательским соглашением http://www.mathnet.ru/rus/agreement

Параметры загрузки:

IP : 54.197 .130 .99

26 апреля 2023 г., 08:52:55 


\section{УСТОЙЧИВОСТЬ ПОЛНОТЫ ЭКСПОНЕНЦИАЛЬНЫХ СИСТЕМ НА ВЫПУКЛЫХ КОМПАКТАХ В $\mathbb{C}$}

\section{Б. Н. Хабибуллин}

Получено достаточное условие устойчивости полноты систем экспонент $\left\{\exp \lambda_{n} z\right\}$ при сдвигах показателей $\lambda_{n}$ в пространствах функций, непрерывных на выпуклом компакте $K \subset \mathbb{C}$ с непустой внутренностью и голоморфных внутри $K$. Это условие - обобщение важного частного случая соответствующего результата Редхеффера и Александера для отрезка.

Библиограффия: 20 названий.

Введение. Все функции и меры, если противное не оговорено или не продиктовано контекстом, предполагаются комплекснозначньми.

Пусть $K$ - компакт на комплексной плоскости $\mathbb{C}$. Как обычно, $C(K)$ - банахово пространство всех непрерьвных функций на $K$ с естественной нормой

$$
\|f\|_{K}=\sup \{|f(z)|: z \in K\}, \quad f \in C(K) .
$$

Через $A(K)$ обозначаем подпространство пространства $C(K)$, состоящее из функций, голоморфных во внутренности Int $K$ компакта $K$, если Int $K \neq \varnothing$, с той же нормой (0.1). В частности, при Int $K=\varnothing$ имеем $A(K)=C(K)$.

Пусть $\Lambda=\left\{\lambda_{n}\right\} \subset \mathbb{C}, n \in \mathbb{N},-$ последовательность комплексных чисел (точек), не имеющая предельных точек в $\mathbb{C}$. Последовательности $\Lambda \subset \mathbb{C}$ сопоставим экспоненциальную систему

$$
\operatorname{Exp} \Lambda=\left\{z^{k-1} e^{\lambda z}: \lambda \in \Lambda, 1 \leqslant k \leqslant \Lambda(\lambda), k \in \mathbb{N}\right\},
$$

где $\Lambda(\lambda)$ - число повторений точки $\lambda$ в последовательности $\Lambda$.

Следуя, например, [1], [2], систему $\operatorname{Exp} \Lambda$ назьваем ${ }^{1}$ полной на компакте $K$, если замькание ее линейной оболочки в пространстве $A(K)$ совпадает с $A(K)$.

В [3, теорема 3] в ослабленной форме, а затем в окончательном виде в обзоре [4, теорема 14] установлена

Работа выполнена при поддержке Российского фонда фундаментальных исследований, грант № 00-01-00770.

${ }^{1} \mathrm{~B}$ ряде работ принято двойственное определение понятия полноты системы функций в топологическом векторном пространстве $E$ или, иначе говоря, относительно $E$, а именно: $E$ - сильн сопряженное к пространству, которому принадлежит система, и аннулирует эту систему только нулевой функционал из $E$ (см., в частности, [3, теорема 3$]$, $[4$, с. 5]). Это иногда приводило к неточностям $[5$, c. 212$]$ или неясностям $[6$, c. 213$]$ в формулировке первоначального варианта исходной для нас теоремы Александера-Редхеффера. 
ТЕОРЕМА АЛЕКСАНДЕРА-РЕДХЕФФЕРА. Если для последовательностей комплексных чисел $\Lambda=\left\{\lambda_{n}\right\}$ и $\Gamma=\left\{\gamma_{n}\right\}, n=1,2, \ldots$, выполнено соотношение

$$
\sum_{n=1}^{\infty} \frac{\left|\lambda_{n}-\gamma_{n}\right|}{1+\left|\operatorname{Re} \lambda_{n}\right|+\left|\operatorname{Re} \gamma_{n}\right|}<+\infty
$$

то $\operatorname{Exp} \Lambda$ и $\operatorname{Exp} \Gamma$ полны или неполны на любом отрезке $K \subset \mathbb{R}$ одновременно.

Привлекательность этой теоремы Александера-Редхеффера как минимум в том, что для каждой последовательности $\Lambda$ и Г по отдельности условие $(0.3)$ не диктует какой-либо регулярности в ее распределении.

Этот результат сразу влечет

СлЕДСТВИЕ. Если последовательности $\Lambda$ и связаны соотношением

$$
\sum_{n=1}^{\infty}\left|\lambda_{n}-\gamma_{n}\right|<+\infty
$$

то системы $\operatorname{Exp} \Lambda$ и $\operatorname{\operatorname {xp}} \Gamma$ полны или неполны на отрезке $K$ в $\mathbb{C}$ только одновременно.

Многие другие результаты об устойчивости полноты экспоненциальных систем для пространств $C[-a, a]$ или $L^{p}(-a, a)$, установленные до 1977 года, отражены в обзоре Редхеффера [4]. Серия тонких результатов в этом направлении принадлежит Седлецкому (см. [6]-[11] и т.д.).

Известные нам работы, связанные с вопросами именно полноты экспоненциальных систем на компакте с непустой внутренностью, немногочисленны (см., например, [12]-[14]). В то же время исследование полноты таких систем на компактах в $\mathbb{C}$ с непустой внутренностью тем более актуально и в определенной степени выдвигается на первый план, так как существование "хороших" базисов или представляющих систем для всего пространства $A(K)$, не говоря уж о таковых именно вида $(0.2)$, проблематично. Так известно, что не существует абсолютно представляющих экспоненциальных систем для $A(K)$ (см. [15]). В $A(K)$, как и в $C[a, b]$, нет безусловных базисов. Более того, весьма вероятно, что в $A(K)$ не существует даже базисов вида $(0.2)$.

В [14, теорема 2] был установлен ослабленньй вариант обобщения теоремы Александера-Редхеффера на выпуклые компакты $K$ с непустой внутренностью, где показано, что при определенном условии "близости" последовательностей $\Lambda$ и $\Gamma$, естественным образом развивающем соотношение $(0.3)$ с учетом геометрии выпуклого компакта $K$, системы $\operatorname{Exp} \Lambda$ и $\operatorname{Exp} \Gamma$ полны или неполны одновременно на $K$ с возможным “зазором" в две экспоненты. Но проблему полного переноса как теоремы Александера-Редхеффера, так и приведенного вьше следствия на произвольные вьпуклые компакты $K \subset \mathbb{C}$ с непустой внутренностью этот результат не решает. Основной результат настоящей статьиследующая теорема об устойчивости полноты, которая в полной мере обобщает следствие на произвольные вьпуклые компакты в $\mathbb{C}$. 
ТЕОРЕМА ОБ УСТоЙЧИвоСТИ ПолноТЫ. Если последовательности $\Lambda$ и Г связаны соотношением (0.4), то системы $\operatorname{Exp} \Lambda$ и $\operatorname{Exp} \Gamma$ могут быть полны на выпуклом компакте в $\mathbb{C}$ только одновременно.

Этот результат был анонсирован ранее частично в [16] и в окончательном виде в [17].

Автор выражает глубокую признательность И.Ф. Красичкову-Терновскому и С.В. Попенову за полезные обсуждения.

1. О сопряженном к $A(K)$. Далее всюду, не оговаривая особо, считаем, что компакт $K$ вьпукльй.

Через $A^{*}(K)$ и $C^{*}(\partial K)$ обозначаем пространства, сильно сопряженные соответственно к $A(K)$ и к пространству $C(\partial K)$ функций, непрерьвных на $\partial K$.

Поскольку каждая функция $f \in A(K)$ однозначно определяется своими значениями на границе и множество сужений функций из $A(K)$ на $\partial K$ образует замкнутое подпространство в пространстве $C(\partial K)$, то по теореме Хана-Банаха каждому линейному непрерывному функционалу $\mathscr{L} \in A^{*}(K)$ с нормой $\|\mathscr{L}\|^{*}$ в $A^{*}(K)$ можно сопоставить линейньй непрерьвньй функционал $1 \in C^{*}(\partial K)$ с той же нормой. По теореме Рисса каждый функционал $\mathbf{l} \in C^{*}(\partial K)$ можно однозначно отождествить с некоторой конечной борелевской мерой на $\partial K$, которую обозначаем тем же символом 1, и для меры 1 на $\partial K$ пишем также $\mathbf{l} \in C^{*}(\partial K)$. Такое сопоставление $\mathscr{L} \rightarrow \mathbf{l}$ отнюдь не однозначно. Оно подчиняется правилу

$$
\mathscr{L}(f)=\int_{\partial K} f(\zeta) d \mathbf{l}(\zeta), \quad f \in A(K) .
$$

Нормой функционала (меры) $1 \in C^{*}(\partial K)$ в $C^{*}(\partial K)$ служит полная вариация $\operatorname{Var}[1]$ меры 1.

В обратном направлении можно сказать, что каждый линейньй непрерьвньй функционал 1 на $C(\partial K)$, рассматриваемьй как мера 1 на $\partial K$, однозначно порождает линейный непрерывный функционал $\mathscr{L} \in A^{*}(K)$ по закону (1.1).

Каждую меру $\mathbf{l}$ на $\partial K$, связанную с функционалом $\mathscr{L} \in A^{*}(K)$ по правилу (1.1), называем далее определяющей мерой функционала $\mathscr{L}$ и говорим, что мера 1 определяет функционал $\mathscr{L}$.

Таким образом, согласно (1.1) для каждой меры 1 , определяющей функционал $\mathscr{L} \in$ $A^{*}(K)$, выполнено

$$
\|\mathscr{L}\|^{*} \leqslant \operatorname{Var}[1]
$$

и, кроме того, для $\mathscr{L} \in A^{*}(K)$ существует мера $1 \in C^{*}(\partial K)$, для которой в (1.2) можно поставить знак равенства.

Нам удобно обозначить через $[0, S]^{\circlearrowleft}$ отрезок $[0, S]$ с отождествленными точками 0 и $S$, т.е. фактически окружность с периметром $S$ с отмеченной на ней начальной точкой 0 с обычной метрикой - длиной кратчайшей дуги, соединяющей точки.

Пусть задана естественная (натуральная) параметризация по длине дуги границы (обход всегда против часовой стрелки) выпуклого компакта $K$

$$
\zeta:[0, S]^{\circlearrowleft} \rightarrow \partial K, \quad S-\text { длина } \partial K, \quad \zeta(0)=\zeta(S)=\zeta_{0} .
$$


Она, очевидно, удовлетворяет условию Липшица

$$
|\zeta(s)-\zeta(t)| \leqslant|s-t|
$$

следовательно, является абсолютно непрерьвной функцией ограниченной вариации на $[0, S]^{\circlearrowleft}$. Кроме того, параметризация $(1.3)$ как непрерывная биекция компакта $[0, S]^{\circlearrowleft}$ на $\partial K$ является гомеоморфизмом между $[0, S]^{\circlearrowleft}$ и $\partial K$.

При заданной параметризации (1.3) каждый функционал $\mathscr{L}$ из $A^{*}(K)$ с определяющей мерой 1 можно задать непрерьвной справа функцией ограниченной вариации $l$ на $[0, S]^{\circlearrowleft}$, где

$$
l(s)=\mathbf{l}(\zeta((0, s])), \quad 0<s \leqslant S, \quad l(0)=0 .
$$

Тогда согласно (1.1)

$$
\mathscr{L}(f)=\int_{0}^{S} f(\zeta(s)) d l(s), \quad f \in A(K),
$$

где интеграл понимается как интеграл Стилтьеса.

Обратно, при заданной параметризации (1.3) каждая непрерьвная справа функция ограниченной вариации $l$ на $[0, S]^{\circlearrowleft}, l(0)=0$, по закону (1.5) однозначно определяет борелевскую меру 1 , которая, в свою очередь, уже однозначно определяет функционал $\mathscr{L} \in A^{*}(K)$ по правилу (1.6) или (1.1). При этом полная вариация $\operatorname{Var}[l]$ функции $l$ на $[0, S]^{\circlearrowleft}$ совпадает с полной вариацией $\operatorname{Var}[1]$ меры 1 , поскольку параметризация (1.3) гомеоморфизм, т.е. "запас" непрерьвных функций на $[0, S]^{\circlearrowleft}$ и на $\partial K$ один и тот же, а нормы соответствующих линейных непрерьвных функционалов задаются как полные вариации соответствующих мер (функций).

Каждую непрерьвную справа функцию ограниченной вариации $l$ на $[0, S]^{\circlearrowleft}, l(0)=0$, связанную при заданной параметризации с функционалом $\mathscr{L} \in A^{*}(K)$ по правилу (1.6), назьваем далее определяющей функиией функционала $\mathscr{L}$ и говорим, что функция $l$ определяет функционал $\mathscr{L}$.

Согласно сказанному вьше об определяющих мерах $1 \in C^{*}(\partial K)$ для каждой функции $l$, определяющей функционал $\mathscr{L} \in A^{*}(K)$, вьполнено

$$
\|\mathscr{L}\|^{*} \leqslant \operatorname{Var}[l]
$$

и, кроме того, для $\mathscr{L} \in A^{*}(K)$ при любой параметризации (1.3) существует непрерывная справа функиия ограниченной вариачии $l$ на $[0, S]^{\circlearrowleft}, l(0)=0$, для которой в (1.7) мохно поставить знак равенства.

Характеристическая функиия (преобразование Лапласа) функционала $\mathscr{L} \in A^{*}(K)$ с определяющей мерой 1 и с определяющей функцией $l$ согласно (1.1) и (1.6) однозначно определяется как целая функция экспоненциального типа

$$
L(z)=\mathscr{L}\left(e^{z \zeta}\right)=\int_{\partial K} \exp (z \zeta) d \mathbf{l}(\zeta)=\int_{0}^{S} \exp (z \zeta(s)) d l(s), \quad z \in \mathbb{C}
$$

В силу полноты системы экспонент $\left\{e^{z \zeta}: z \in \mathbb{C}\right\}, \zeta \in K$, в $A(K)$ если $L$ - нулевая функция, то функционал $\mathscr{L}$ также нулевой. 


\section{2. Конструкция Левинсона-Александера-Редхеффера для выпуклого} компакта. В принятых в предыдущем пункте соглашениях и обозначениях (1.3)-(1.8) справедлив следуюший вариант тождества Левинсона, применявшийся им для случая отрезка $K \in \mathbb{R}[18]$.

ПРЕДЛОЖенИЕ 1. Если $L$ - характеристическая функиия функиионала $\mathscr{L} \in$ $A^{*}(K)$ c определяющей функцией l и L обращается в нуль в точке $\lambda$, то для функиионала $\mathscr{L}_{\lambda} \in A^{*}(K)$ с характеристической функиией $L_{\lambda}=L(z) /(z-\lambda)$ можно выбрать определяющую функиию $l_{\lambda}$ (при той же параметризачии (1.3), что и для l) с плотностью распределения

$$
d l_{\lambda}(s)=-\left(\int_{0}^{s} e^{\lambda(\zeta(t)-\zeta(s))} d l(t)\right) d \zeta(s)=\left(\int_{s}^{S} e^{\lambda(\zeta(t)-\zeta(s))} d l(t)\right) d \zeta(s) .
$$

ДокАЗАТЕЛЬСтво. Перепишем характеристическую функцию $L$ в виде

$$
L(z)=\int_{0}^{S} e^{(z-\lambda) \zeta(s)} e^{\lambda \zeta(s)} d l(s)=\int_{0}^{S} e^{(z-\lambda) \zeta(s)} d \int_{0}^{s} e^{\lambda \zeta(t)} d l(t),
$$

откуда интегрированием по частям ${ }^{2}[19$, гл. VIII $]$ с учетом $L(\lambda)=0$ получаем

$$
\begin{aligned}
L(z) & =e^{(z-\lambda) \zeta(s)} \int_{0}^{S} e^{\lambda \zeta(t)} d l(t)-\int_{0}^{S}\left(\int_{0}^{s} e^{\lambda \zeta(t)} d l(t)\right) d e^{(z-\lambda) \zeta(s)} \\
& =e^{(z-\lambda) \zeta(S)} L(\lambda)-\int_{0}^{S}\left(\int_{0}^{s} e^{\lambda \zeta(t)} d l(t)\right)(z-\lambda) e^{(z-\lambda) \zeta(s)} d \zeta(s) \\
& =-(z-\lambda) \int_{0}^{S} e^{z \zeta(s)}\left(\int_{0}^{s} e^{\lambda(\zeta(t)-\zeta(s))} d l(t)\right) d \zeta(s) .
\end{aligned}
$$

Поделив полученное равенство на $z-\lambda$ и выбирая $l_{\lambda}(s)$ как в $(2.1)$, в силу однозначной определенности функционала его характеристической функцией получим (2.1). Функционал $\mathscr{L}_{\lambda}$ с определяющей функцией $l_{\lambda}$ принадлежит $A^{*}(K)$, так как согласно конструкции $(2.1)$ функция $l_{\lambda}$ ограниченной вариации на $[0, S]^{\circlearrowleft}$. Независимость от $\zeta_{0}$ видна по построению. Последнее соотношение в (2.1) вытекает из тождества

$$
L(\lambda)=\int_{0}^{s} e^{\lambda \zeta(t)} d l(t)+\int_{s}^{S} e^{\lambda \zeta(t)} d l(t) \equiv 0 .
$$

ПРЕДЛОЖЕНИЕ 2. В условиях и обозначениях предложения 1 для функиионала $\mathscr{G} \in A^{*}(K)$ с характеристической функиией

$$
G(z)=\frac{z-\gamma}{z-\lambda} L(z)
$$

можно подобрать определяющей функиию $g$, связанную $\mathrm{cl}$ соотношением

$$
d g(s)=d l(s)+(\lambda-\gamma) d l_{\lambda}(s) .
$$

\footnotetext{
${ }^{2} \mathrm{~B}$ книге Натансона большинство утверждений доказаны для функций и мер со значениями на $[-\infty,+\infty]$, но они мгновенно переносятся на функции и меры со значениями в $\mathbb{C} \cup\{\infty\}$, если рассмотреть отдельно мнимые и действительные их части.
} 
ДоКАЗАТЕЛЬСТво. Достаточно воспользоваться тождеством

$$
G(z)=L(z)+(\lambda-\gamma) \frac{L(z)}{z-\lambda}
$$

и однозначной определенностью функционала его характеристической функцией. Принадлежность $\mathscr{G}$ к $A^{*}(K)$ следует из вида его определяющей функции $(2.5)$.

Следующее утверждение распространяет соответствующую оценку из [3] и $[4$, п. 6$]$ для отрезка на выпуклый компакт.

ПРЕДЛОЖЕНИЕ 3. Если характеристическая функиия $L$ ненулевого функиионала $\mathscr{L} \in A^{*}(K)$ удовлетворяет условию $L(\lambda)=0$, то функиия $G$ из (2.4) - характеристическая функция некоторого ненулевого функционала $\mathscr{G} \in A^{*}(K)$ u

$$
\|\mathscr{G}-\mathscr{L}\|^{*} \leqslant S \cdot|\lambda-\gamma| \cdot\|\mathscr{L}\|^{*}
$$

где $S$ - периметр выпуклого компакта $K$.

ДоКАЗАТЕЛЬСТво. Используя сдвиг всей плоскости $\mathbb{C}$ и поворот ее на соответствующий угол, можно считать, не умаляя общности, что точка $\lambda$ лежит на положительной полуоси $(0,+\infty)$.

Как отмечалось в п. 1, можно подобрать определяющую меру $1 \in A^{*}(K)$ функционала $\mathscr{L}$ так, что

$$
\|\mathscr{L}\|^{*}=\operatorname{Var}[\mathbf{l}] .
$$

Опишем теперь выбор параметризации (1.3) (см. рис. 1).

Пусть $l_{+}$и $l_{-}$- две опорные прямые компакта $K$, ортогональные положительной полуоси, и $l_{-}$расположена левее $l_{+}$. В качестве начальной точки $\zeta_{0}=\zeta(0)$ в параметризации (1.3) выберем одну из точек опоры опорной прямой $l_{-}$. Отметим также далее одну из точек опоры $\zeta_{+}=\zeta\left(s_{+}\right)$опорной прямой $l_{+}$.

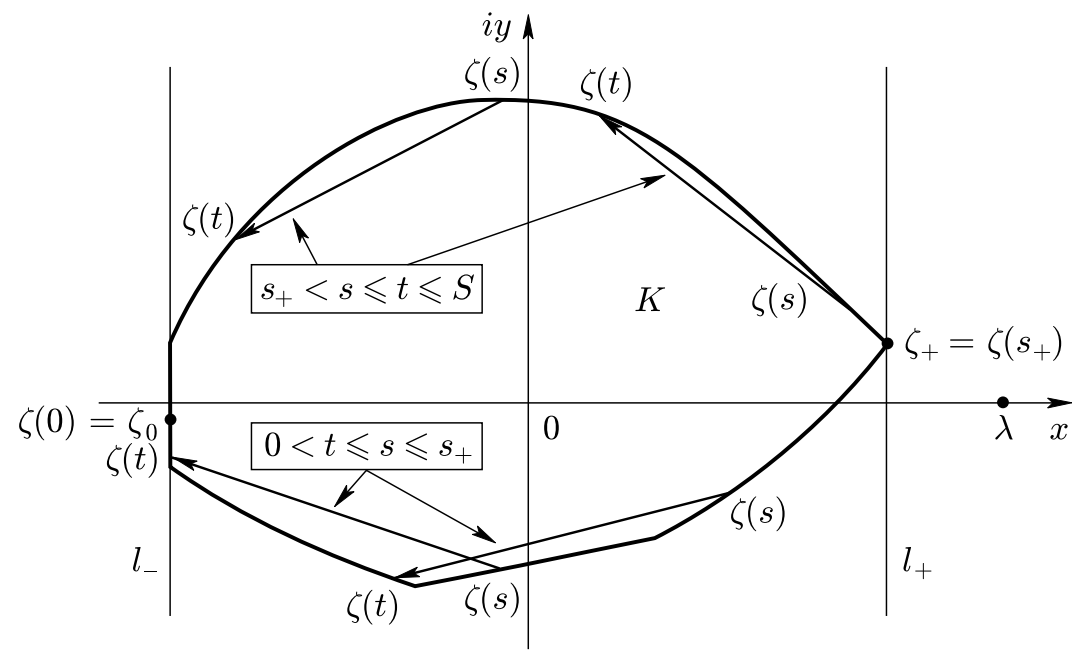

Рис. 1. Выбор параметризации (обход против часовой стрелки) 
При выбранной параметризации согласно (2.7) по мере 1 можно построить определяющую функцию $l$ такую, что

$$
\|\mathscr{L}\|^{*}=\operatorname{Var}[l] .
$$

Тогда по предложениям 2 и $1 G$-характеристическая функция для некоторого функционала $\mathscr{G} \in A^{*}(K)$, для которого можно подобрать определяющую функцию $g$ (при той же параметризации) так, что

$$
\begin{aligned}
d(g-l)(s) & =(\lambda-\gamma)\left(-\int_{0}^{s} e^{\lambda(\zeta(t)-\zeta(s))} d l(t)\right) d \zeta(s) \\
& =(\lambda-\gamma)\left(\int_{s}^{S} e^{\lambda(\zeta(t)-\zeta(s))} d l(t)\right) d \zeta(s) .
\end{aligned}
$$

Отсюда

$$
\operatorname{Var}[g-l] \leqslant|\lambda-\gamma| \sup _{s} \min \left\{\left|\int_{0}^{s} e^{\lambda(\zeta(t)-\zeta(s))} d l(t)\right|,\left|\int_{s}^{S} e^{\lambda(\zeta(t)-\zeta(s))} d l(t)\right|\right\} \cdot \operatorname{Var}[\zeta],
$$

где вариация $\operatorname{Var}[\zeta]$ параметризации (1.3) есть периметр $S$. Продолжая эту оценку, получаем

$$
\operatorname{Var}[g-l] \leqslant|\lambda-\gamma| \sup _{s} \min \left\{B_{0}(s), B_{S}(s)\right\} \cdot \operatorname{Var}[l] \cdot S,
$$

где

$$
B_{0}(s)=\sup _{0<t \leqslant s}\left|e^{\lambda(\zeta(t)-\zeta(s))}\right|, \quad B_{S}(s)=\sup _{s<t \leqslant S}\left|e^{\lambda(\zeta(t)-\zeta(s))}\right|,
$$

или иначе

$$
\begin{aligned}
& B_{0}(s)=\exp \left(\lambda \sup _{0<t \leqslant s} \operatorname{Re}(\zeta(t)-\zeta(s))\right) \\
& B_{S}(s)=\exp \left(\lambda \sup _{s<t \leqslant S} \operatorname{Re}(\zeta(t)-\zeta(s))\right)
\end{aligned}
$$

Из рис. 1 легко видеть, что для выпуклого компакта $K$ справедливы оценки

$$
\begin{array}{ll}
\operatorname{Re}(\zeta(t)-\zeta(s)) \leqslant 0 & \text { при всех } 0<t \leqslant s \leqslant s_{+}, \\
\operatorname{Re}(\zeta(t)-\zeta(s)) \leqslant 0 & \text { при всех } s_{+}<s \leqslant t \leqslant S .
\end{array}
$$

Отсюда согласно (2.10) и (2.11) соответственно имеем

$$
\begin{array}{ll}
B_{0}(s) \leqslant 1 & \text { при всех } 0<t \leqslant s \leqslant s_{+}, \\
B_{S}(s) \leqslant 1 & \text { при всех } s_{+}<s \leqslant t \leqslant S .
\end{array}
$$

Применяя последние оценки к (2.9), получаем

$$
\operatorname{Var}[g-l] \leqslant|\lambda-\gamma| \cdot 1 \cdot S \cdot \operatorname{Var}[l] .
$$

Отсюда, поскольку $g-l$ - определяюшая функция для функционала $\mathscr{G}-\mathscr{L}$, имеем

$$
\|\mathscr{G}-\mathscr{L}\|^{*} \leqslant S \cdot|\lambda-\gamma| \cdot \operatorname{Var}[l]=S \cdot|\lambda-\gamma| \cdot\|\mathscr{L}\|^{*},
$$

где последнее равенство обеспечено выбором (2.8) определяющей функции $l$. Предложение доказано. 
3. О сходимости в $A^{*}(K)$. Следующее утверждение в целом известно и применялось в [3] в конкретной ситуации, но в вычлененном виде нам не встречалось. Поэтому мы приводим его с доказательством.

ПРЕДЛОЖЕНИЕ 4. Пусть $\left\{x_{n}\right\}$ - последовательность в нормированном пространстве $X$ с нормой $\|$ · $\|$. Если последовательность неотрицательных чисел $\left\{d_{n}\right\}$ такова, что

$$
\sum_{n} d_{n}<+\infty
$$

$u$

$$
\left\|x_{n+1}-x_{n}\right\| \leqslant d_{n}\left\|x_{n}\right\|, \quad n=1,2, \ldots,
$$

то последовательность $\left\{x_{n}\right\}$ фундаментальная.

ДоКАЗАТЕЛЬСТВо. Из соотношений (3.2) следует

$$
\left(1-d_{n}\right)\left\|x_{n}\right\| \leqslant\left\|x_{n+1}\right\| \leqslant\left(1+d_{n}\right)\left\|x_{n}\right\| .
$$

Правая часть этого неравенства вместе с (3.1) влечет

$$
\left\|x_{n+1}\right\| \leqslant \prod_{k=1}^{n}\left(1+d_{k}\right)\left\|x_{1}\right\| \leqslant \exp \left(\sum_{k} d_{k}\right)\left\|x_{1}\right\| \leqslant M
$$

где $M$ - постоянная.

Из левой части (3.3) при $n \geqslant m$ следует

$$
\left\|x_{n}-x_{m}\right\| \leqslant\left\|x_{n}-x_{n-1}\right\|+\cdots+\left\|x_{m+1}-x_{m}\right\| \leqslant\left(d_{n-1}+d_{n-2}+\cdots+d_{m}\right) M,
$$

откуда в силу сходимости ряда (3.1) получаем фундаментальность последовательности $\left\{x_{n}\right\}$.

Приведем вариацию предложения 4 для $A^{*}(K)$.

ПРЕДЛОЖЕНИЕ 5. Если $\left\{\mathscr{L}_{n}\right\}$ - последовательность функиионалов из $A^{*}(K) c$ характеристическими функииями $L_{n}$ и для последовательности $\left\{d_{n}\right\}$, удовлетворяющей (3.1), выполнены оценки

$$
\left\|\mathscr{L}_{n+1}-\mathscr{L}_{n}\right\|^{*} \leqslant d_{n}\left\|\mathscr{L}_{n}\right\|, \quad n=1,2, \ldots,
$$

то последовательность функиионалов $\mathscr{L}_{n}$ сходится в $A^{*}(K)$ к некоторому функиионалу $\mathscr{G} \in A^{*}(K)$ с характеристической функиией $G$, а последовательность иельх функиий $L_{n}$ сходится к функиии $G$ по норме

$$
\|L\|_{K}^{\star}=\sup _{z \in \mathbb{C}} \frac{|L(z)|}{\exp (k(-\arg z)|z|)}
$$

где $L$ - произвольная характеристическая функиия функиионала из $A^{*}(K), k$ опорная функиия выпуклого компакта $K$.

В частности, если предел последовательности иелых функиий $L_{n}$ в топологии равномерной сходимости на компактах - ненулевая целая функция, то функционал $\mathscr{G} \in A^{*}(K)$ ненулевой. 
ДоКАЗАТЕЛЬСТВо. По условию (3.4) и по предложению 4 последовательность $\mathscr{L}_{n}$ фундаментальная в $A^{*}(K)$. Пространство, сильно сопряженное к нормированному, банахово. Значит, эта последовательность сходится в $A^{*}(K)$ к некоторому функционалу $\mathscr{G} \in A^{*}(K)$. По определению (1.8) имеем $\left|L_{n}(z)-G(z)\right|=\left|\left(\mathscr{L}_{n}-\mathscr{G}\right)\left(e^{z \zeta}\right)\right|$. Отсюда ввиду (1.8) для некоторой определяющей меры $\mathbf{m}_{n} \in C^{*}(\partial K)$ функционала $\mathscr{L}_{n}-\mathscr{G}$ такой, что $\operatorname{Var}\left[\mathbf{m}_{n}\right]=\left\|\mathscr{L}_{n}-\mathscr{G}\right\|^{*}$, получаем

$$
\begin{aligned}
\left|L_{n}(z)-G(z)\right| & \leqslant \operatorname{Var}\left[\mathbf{m}_{n}\right] \cdot \sup _{\zeta \in K}|\exp (z \zeta)| \\
& =\left\|\mathscr{L}_{n}-\mathscr{G}\right\|^{*} \cdot \sup _{\zeta \in K} \exp (\operatorname{Re}(z \zeta))=\left\|\mathscr{L}_{n}-\mathscr{G}\right\|^{*} \cdot k(-\arg z)|z| .
\end{aligned}
$$

Это устанавливает сходимость по норме (3.5) для характеристических функций. Эта сходимость сильнее, чем равномерная сходимость на компактах, откуда следует заключительное утверждение.

4. Доказательство теоремы об устойчивости полноты. В силу симметричности условия (0.4) по $\Lambda$ и $Г$ достаточно доказать, что неполнота $\operatorname{Exp} \Lambda$ на $K$ влечет неполноту $\operatorname{Exp} \Gamma$ на $K$.

Если система $\operatorname{Exp} \Lambda$ неполна на $K$, то по теореме Хана-Банаха найдется ненулевой функционал $\mathscr{L} \in A^{*}(K)$, аннулируюший систему $\operatorname{Exp} \Lambda$. После преобразования Лапласа (1.8) это означает, что ненулевая характеристическая функция $L$ обращается в нуль на последовательности $\Lambda$ в том смысле, что кратность нуля функции $L$ в каждой точке $\lambda \in \mathbb{C}$ не меньше числа повторений точки $\lambda$ в последовательности $\Lambda$.

Рассмотрим последовательность целых функций $L_{n}, n=1,2, \ldots$, построенную рекуррентно по следующему правилу:

$$
L_{1}=L, \quad L_{n+1}(z) \equiv \frac{z-\gamma_{n}}{z-\lambda_{n}} L_{n}(z), \quad z \in \mathbb{C} .
$$

Легко показать, что последовательность целых функций $L_{n}$ равномерно на компактах сходится к ненулевой целой функции $G$, обращающейся в нуль на последовательности $\Gamma$.

Конструкция каждой пары целых функций $L_{n}$ и $L_{n+1}$ точно такая же, как пары $L$ и $G$ в предложении $3, \lambda=\lambda_{n}, \gamma=\gamma_{n}$. Тогда по предложению 3 вьполнены оценки (3.4) для $d_{n}=S \cdot\left|\lambda_{n}-\gamma_{n}\right|$. По условию (0.4) ряд (3.1) сходится. По предложению 5 последовательность функционалов $\mathscr{L}_{n}$ сходится в $A^{*}(K)$ к функционалу $\mathscr{G} \in A^{*}(K)$, аннулирующему систему $\operatorname{Exp} \Gamma$, с характеристической функцией $G$. Эта функция ненулевая, так как сходимость по норме (3.5) сильнее равномерной сходимости на компактах в $\mathbb{C}$. Значит, $\mathscr{G}$ - ненулевой функционал из $A^{*}(K)$. Теорема доказана.

ЗАмЕчАниЕ. Модифицируя примеры Седлецкого [7, с. 805] и Петерсона [20] для отрезка, можно показать, что в теореме об устойчивости полноты, вообще говоря, нельзя заменить условие (0.4) на условие $\left|\lambda_{n}-\gamma_{n}\right| \rightarrow 0, n \rightarrow \infty$, или на условие

$$
\sum_{n} w(n)\left|\lambda_{n}-\gamma_{n}\right|<+\infty
$$

с какой-либо последовательностью $w(n)>0$ такой, что $\inf \{w(n)\}=0$. Построение таких примеров достаточно трудоемко, и здесь мы на этом не останавливаемся. 


\section{СПИСОК ЦИТИРОВАННОЙ ЛИТЕРАТУРЫ}

[1] Левин Б. Я. Распределение корней целых функций. М.: Физматгиз, 1956.

[2] Levin B. Ya. Lectures on Entire Functions. Transl. Math. Monographs. V. 150. Providence (R.I.): Amer. Math. Soc., 1996.

[3] Alexander W.,O., Redheffer R. M. The excess of sets of complex exponentials // Duke Math. J. 1967. V. 34. №1. P. 59-72.

[4] Redheffer R. M. Completeness of sets of complex exponentials // Adv. Math. 1967. V. 24. P. 1-62.

[5] Elsner J. Zulässige Abänderung von Exponentialsystemen im $L^{p}(-A, A) / /$ Math. Z. 1971. V. 120. № 3. P. 211-220.

[6] Седлецкий А. М. Об устойчивости полноты и минимальности в $L^{2}$ системы показательных функций // Матем. заметки. 1974. Т. 15. № 1. С. 213-219.

[7] Седлецкий А. М. Избытки систем показательных функций // Матем. заметки. 1977. Т. 22. № 6. C. $803-814$.

[8] Седлецкий А. М. Избытки систем экспоненциальных функций // Изв. АН СССР. Сер. матем. 1980. Т. 44. №1. С. 203-218.

[9] Седлецкий А. М. Избытки близких систем экспонент в $L^{p} / /$ Сиб. матем. ж. 1983. Т. 24. № 4. C. 164-175.

[10] Седлецкий А. М. Избытки систем показательных функций // Сиб. матем. ж. 1985. Т. 26. № 4. C. 151-158.

[11] Седлецкий А. М. О целых функциях класса С. Н. Бернштейна, не являющихся преобразованиями Фурье-Стилтьеса // Матем. заметки. 1997. Т. 61. №3. С. 367-379.

[12] Напалков В. В. Аппроксимация функций многих переменных с учетом роста коэффициентов аппроксимирующих агрегатов // Матем. сб. 1980. Т. 111. № 1. С. 144-156.

[13] Шилова Г. Н. Аппроксимация линейньми комбинациями экспонент с ограничениями на коэффициенты. Дисс.... к.ф.-м.н. Уфа, 1992.

[14] Хабибуллин Б. Н. Об устойчивости последовательностей неединственности и полноты систем экспонент // Актуальные проблемы математического анализа. Ростов-на-Дону: Изд-во ГинГо, 2000. С. 156-164.

[15] Коробейник Ю.Ф. О некоторых свойствах абсолютно представляющих систем // Линейные операторы в комплексном анализе. Ростов-на-Дону: РГУ, 1994. С. 58-65.

[16] Хабибуллин Б. Н. Об устойчивости полноты систем экспонент в выпуклом компакте // Материалы Международной конференции "Актуальные проблемы математики и механики", посвященной 40-летию мехмата КГУ. Т. 5. Казань: КГУ, 2000. С. 213-214.

[17] Khabibullin B. N. Stability of completeness and minimality of system of complex exponentials // Материалы Международного научного семинара-совещания "Методы функционального анализа и теории функций в различных задачах математической физики”. Уфа: БашГУ, ИМВЦ УНЦ РАН, 2000. С. 72.

[18] Levinson N. Gap and Density Theorem. Amer. Math. Soc. Publ. V. 26. Providence (R.I.): Amer. Math. Soc., 1940.

[19] Натансон И. П. Теория функций вещественной переменной. М.: Наука, 1974.

[20] Peterson D. The excess of sets of complex exponentials // Proc. Amer. Math. Soc. 1974. V. 44. № 2. P. 321-325.

Башкирский государственный университет, г. Уфа

Поступило

E-mail: algeom@bsu.bashedu.ru, khabib-bulat@mail.ru

28.11 .2000

Исправленный вариант

20.11.2001 